\title{
ELECTROHYDRODYNAMIC ENHANCED TRANSPORT AND TRAPPING OF AIRBORNE PARTICLES TO A MICROFLUIDIC AIR-LIQUID INTERFACE
}

\author{
N. Sandström, T. Frisk, G. Stemme, and W. van der Wijngaart \\ Microsystem Technology Lab, School of Electrical Engineering \\ $\mathrm{KTH}$ - Royal Institute of Technology, Stockholm, Sweden
}

\begin{abstract}
We introduce a novel approach for greatly improved transport and trapping of airborne sample to a microfluidic analysis system by integrating an electrohydrodynamic (EHD) air pump with a microfluidic air-liquid interface. In our system, a negative corona discharge partially ionizes the air around a sharp electrode tip while the electrostatic field accelerates airborne particles towards an electrically grounded liquid surface, where they absorb. The air-liquid interface is fixated at the microscale pores of a perforated silicon diaphragm, each pore functioning as a static Laplace valve. Our system was experimentally tested using airborne smoke particles of ammonium chloride and aqueous salt solution as the liquid. We measured that EHD enhanced transport of the particles from the air into the liquid is enhanced over 130 times compared to passive trapping.
\end{abstract}

\section{INTRODUCTION}

Detection, monitoring, and public surveillance of airborne particles and molecules are of great importance for today's security, health care, environment, industrial and military monitoring activities. Particles of particular interest are chemical compounds such as explosives, drugs and toxins, different pollutants and contaminants, and pathogens e.g. bacteria, virus and fungi. Sample-to-liquid interfacing is required in all systems where chemical analysis of airborne particles and substances is performed in liquid environment. Analysis of liquid aggregates is by far the most common analysis form, but the substance of interest is often airborne. Previously shown air sample interfaces for $\mu \mathrm{TAS}$ applications rely on passive trapping of particles to a free liquid surface $[1,2]$ or to a solid surface which can be washed with liquid to collect particles [3, 4].

It has been known for decades that active transport of airborne particles can efficiently be achieved by using corona systems [5]. A large positive or negative voltage $(\mathrm{kV})$ is applied to a high-curvature solid corona electrode which is located opposite to a low-curvature solid collector electrode. The collector is set to ground or a low voltage and an electrostatic field is created between the two solid objects. The high field at the corona electrode causes avalanche breakdown, leading to generation of free electrons and ions. Ions of opposite charge to the corona electrode are accelerated in the electric field towards the collector electrode. Upon collision between traveling ions and neutral molecules and particles, momentum is transferred leading to a drag force on the bulk air towards the collector. Hence, there is a net movement of airborne molecules and particles from the solid corona electrode towards the collector electrode.

Several applications have been shown using different corona systems, e.g. air precipitators [6], dehumidifiers [7], thrusters [8], and cooling devices for electronics [9]. Miniaturized corona systems have been reported in [10, 11], and a system for capturing sub- $\mu \mathrm{m}$ bioparticles in air, such as virions, was shown in [12].

The objective of this work was to actively enhance and promote transport of airborne particles to a liquid interface of e.g. a microfluidic system, by using a liquid based collector electrode. In addition to transportation, the aim was also to study the absorption of the particles to the liquid, for further analysis in the liquid environment.

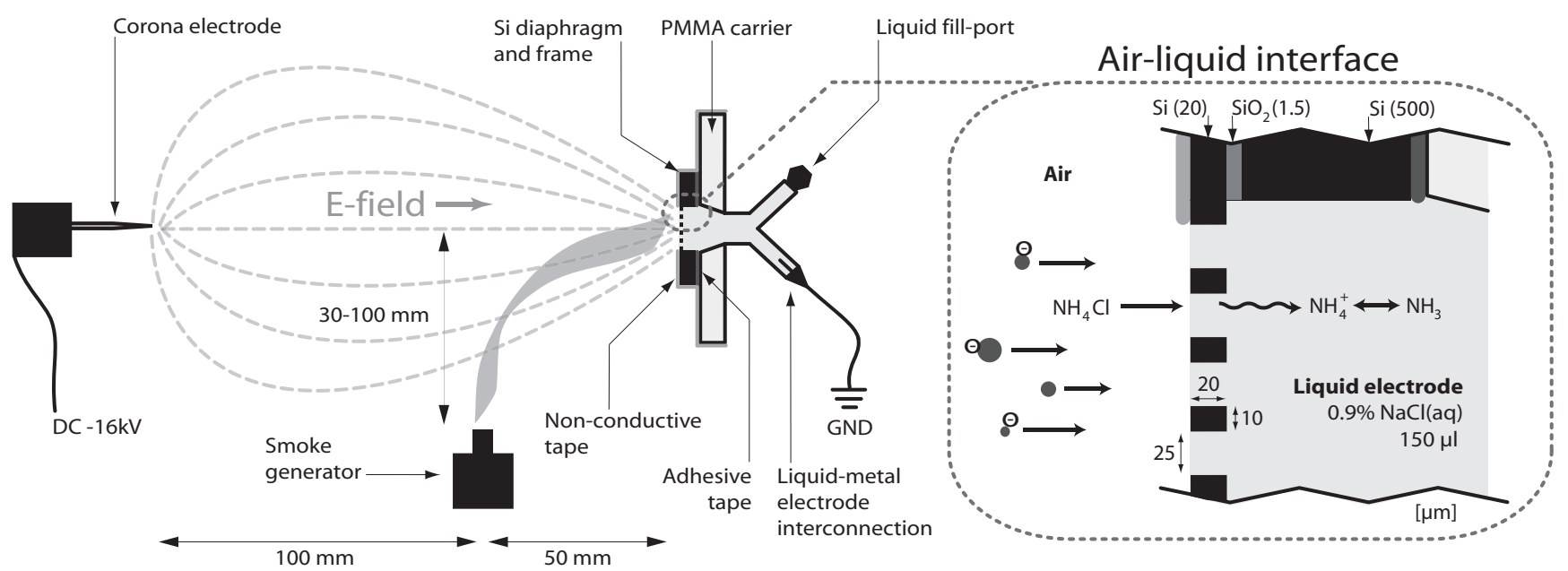

Figure 1: a schematic of the experimental setup. The corona electrode ionizes surrounding air molecules and particles through a high voltage corona discharge. The charged species accelerate in the electric field and drag along uncharged particles towards the liquid collector electrode. When they reach the air-liquid interface they impact and become absorbed. Generated smoke particles were used for visualization and quantification. 


\section{DESIGN AND FABRICATION}

To promote active transportation of airborne particles to an air-liquid interface, we developed a corona system where the conventional solid collector electrode is replaced with a liquid electrode, see figure 1 . The liquid electrode, consisting of an aqueous salt solution, is used together with a corona needle electrode in air. Upon applied corona discharge voltage, airborne molecules and particles are accelerated towards the air-liquid interface of the liquid based collector where they impact.

In this system, a perforated silicon diaphragm is employed to sustain a stable air-liquid interface. Previous work has successfully reported how such diaphragm design was used in an electronic nose to sense narcotics [2]. The diaphragm contains 18,000 perforations, separated by $10 \mu \mathrm{m}$ and each with a diameter of $25 \mu \mathrm{m}$, distributed over a total area of $20 \mathrm{~mm}^{2}$. The bulk silicon gives the diaphragm mechanical stability while surface tension ensures a stable air-liquid interface, i.e. each pore functions as a static Laplace valve. This design offers good tolerance against pressure fluctuations, both unintended and intended such as when refilling or rinsing the system; it discriminates large particles from entering the liquid domain; and keeps the air-liquid interface at a defined position with respect to the liquid container. The diaphragm is fabricated from a silicon-on-insulator (SOI) wafer with standard micromachining techniques, described in [2].

A liquid container in conjunction with the interface is fabricated in plastics, with an electrically grounded liquidmetal wire interconnection and a liquid fill port. The system contains $150 \mu \mathrm{l}$ of liquid $(0.9 \% \mathrm{w} / \mathrm{v}$ sodium chloride in DI water). It is attached to the backside of a carrier substrate of polymethyl methacrylate (PMMA). The silicon diaphragm is attached to the front side of the carrier by double-sided adhesive tape and covered by nonconductive tape, only exposing the perforated air-liquid interface. The interface is aligned with the liquid
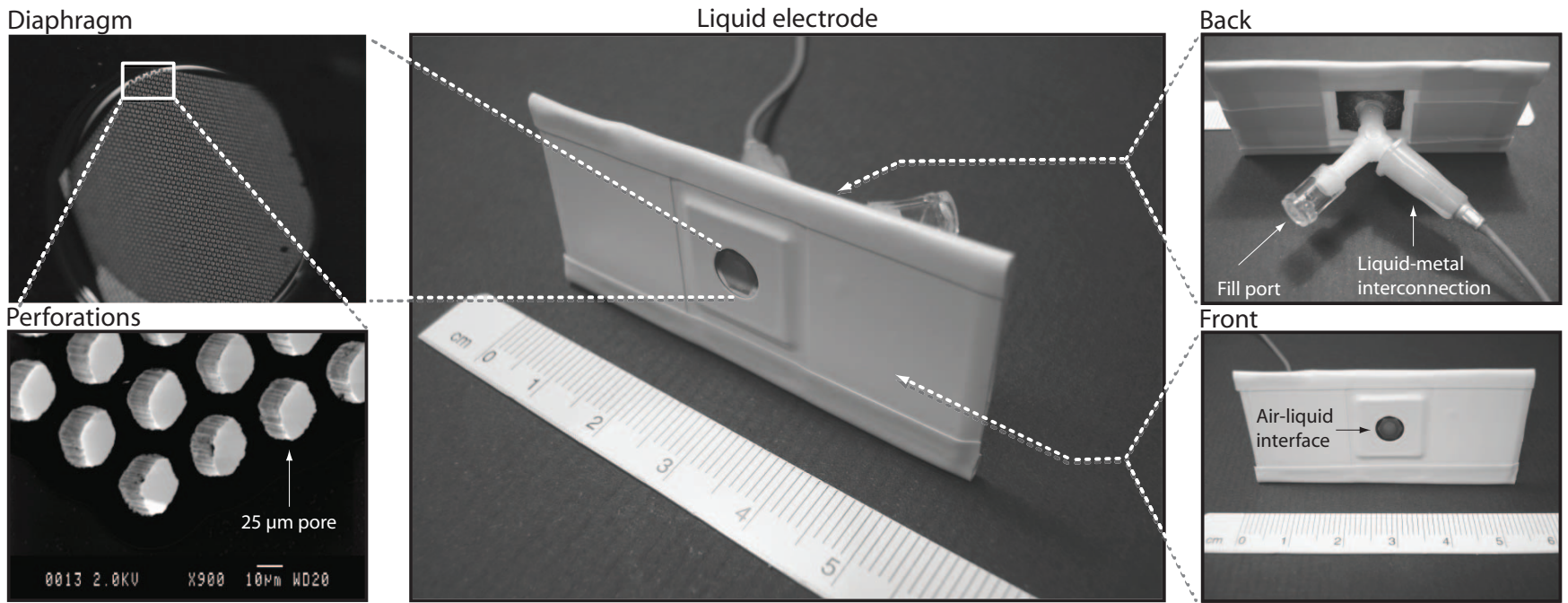

Figure 2: Middle: photograph of the liquid electrode and its package. Left: the silicon component consisting of a rigid frame which holds a perforated diaphragm, adapted from [1]. Right top: the liquid side of the diaphragm is connected to a plastic tube, containing $150 \mu \mathrm{l} 0.9 \% \mathrm{w} / \mathrm{v} \mathrm{NaCl(aq)}$ in direct contact with the grounded metal electrode. Right down: the frame of the interface is covered with non-conductive tape to ensure only exposing the diaphragm as the collector electrode area. 
A negative corona is generated by applying a DC voltage of $U=-16 \mathrm{kV}$ to the corona electrode. Subsequentially, the smoke source is triggered to generate smoke. One shot lasts for $30 \mathrm{~s}$ and the movement of the smoke towards the air-liquid interface is visually inspected. When ammonium chloride smoke is used, $\mathrm{pH}$ measurements are conducted on the solution in the liquid container.

\section{RESULTS}

The active transport of airborne smoke particles towards the air-liquid interface was successfully visualized. Figure 3 shows a fine laminar flow of warm smoke rising upwards due to natural convection when the corona system was turned off. When a voltage was applied and a corona discharge was generated, the smoke was accelerated towards the liquid collector electrode where the particles impact at the interface. The flow speed of the accelerated smoke was visually estimated to approximately $7 \mathrm{~cm} / \mathrm{s}$.

The air-liquid interface was visually inspected after each test. In figure 4, a non-used collector device is shown and compared to devices that have been exposed to the different types of smoke. As anticipated, the ammonium chloride was absorbed and dissolved in the liquid while stearic acid did not dissolve but stayed adsorbed at the interface.

The active transport of airborne particles into the liquid was verified by measuring a shift of acidity in the liquid caused by absorbed particles of ammonium chloride according to (1), see table 1 . In the presence of smoke but in the absence of a corona enhanced transport, no change in acidity could be measured after 30 s (i.e. one shot of smoke). If the exposure of smoke was increased ten times to $300 \mathrm{~s}$ (i.e. ten shots of smoke), a shift of acidity from $\mathrm{pH} 4.5$ to almost 4.0 was measured in the liquid. This corresponds to approximately $0.1 \mathrm{~g}$ of ammonium chloride particles being absorbed into the liquid (calculation based on $\mathrm{K}_{\mathrm{a}}=5.6 \cdot 10^{-10}$ for $\mathrm{NH}_{4}{ }^{+}$). When a corona voltage was applied to the system to enhance transportation and trapping of the airborne particles, a shift from $\mathrm{pH} 4.5$ to 3.5 was measured within only $30 \mathrm{~s}$ of exposure (i.e. 1 shot of smoke). This shift corresponds to absorption of approximately $1.3 \mathrm{~g}$ of smoke particles into the liquid.
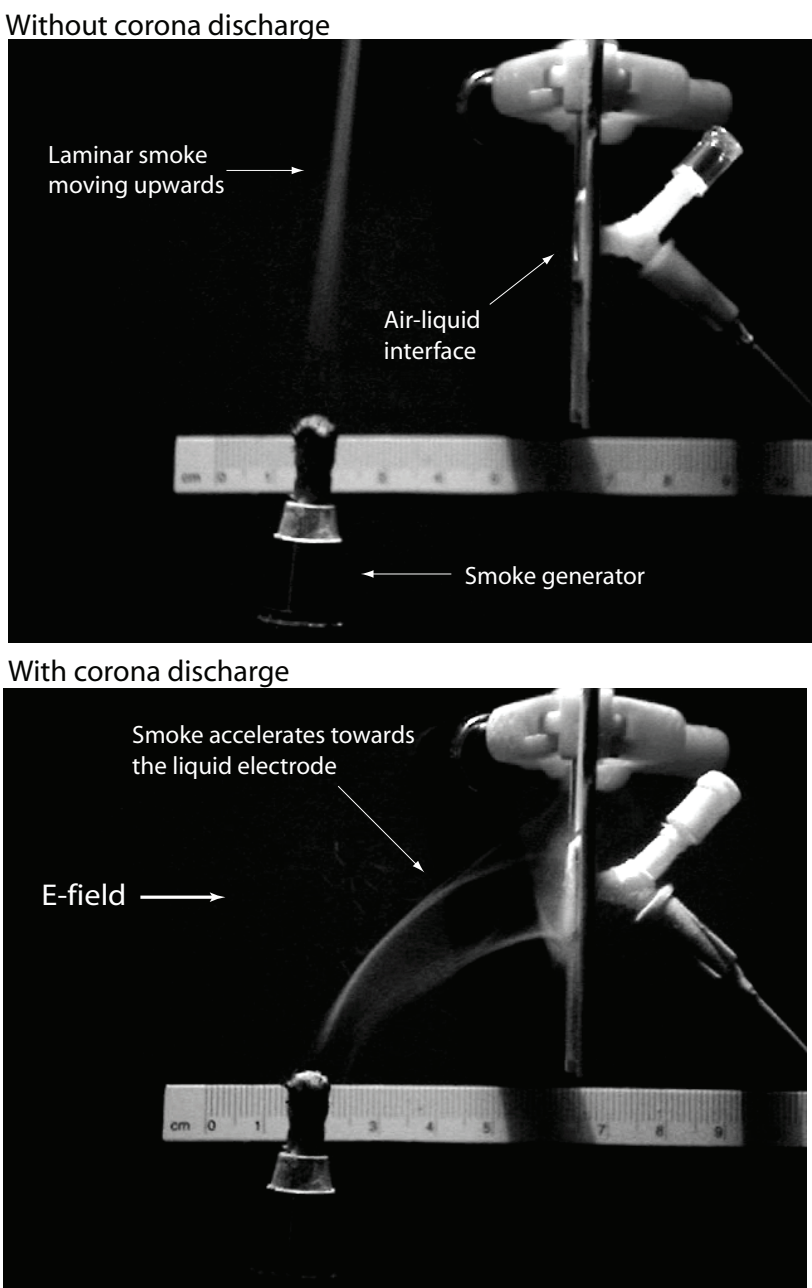

Figure 3: frames from video recordings with EHD promoted transport and trapping. Left: without corona discharge, the smoke is moving straight up. Right: with corona discharge, the smoke is accelerated towards the air-liquid interface. Note: the corona needle electrode is outside the picture frame on the left side.

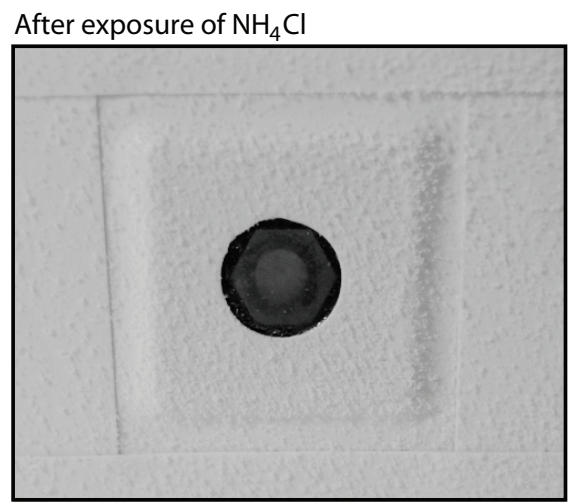

Air-liquid interface, non-used

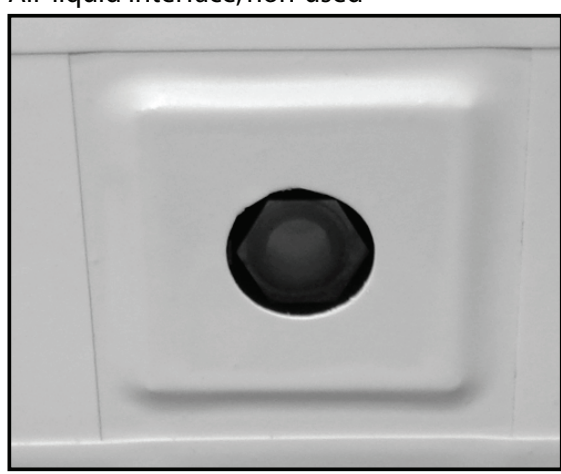

Figure 4: photographs of the air-liquid interface. Left: before usage. Middle: after exposure to smoke of ammonium chloride; the particles dissolve in the liquid and no remains are left at the interface. Right: after exposure to smoke of stearic acid; the particles of the fatty acid, which are insoluble in the liquid, remain at the interface. A concentration gradient of particles in a radial pattern towards the exposed air-liquid interface is visible.

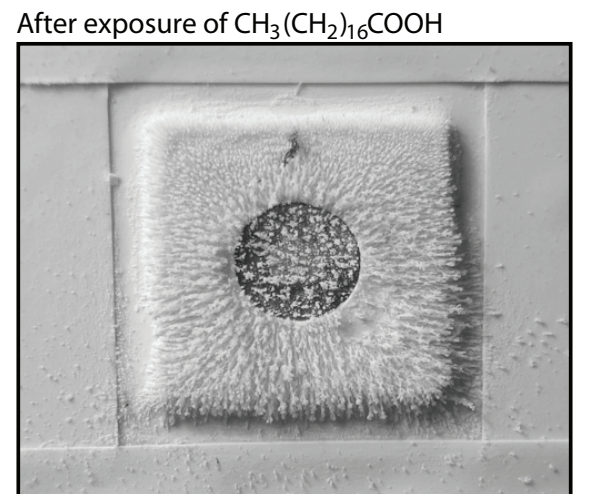


Thus, transportation and trapping of airborne molecules to an air-liquid interface are estimated to be over 130 times more efficient when using the reported corona system compared to a passive system.

Table 1: shift in acidity in the collector liquid measured in pH and calculated mass of absorbed smoke particles for different times of exposure to smoke, with and without an applied corona discharge voltage.

\begin{tabular}{|l|l|l|l|l|}
\hline \multirow{2}{*}{$\begin{array}{l}\text { Exposure } \\
\text { time }\end{array}$} & \multicolumn{2}{|c|}{ Corona off } & \multicolumn{2}{|c|}{ Corona on } \\
$\mathrm{pH}$ & Calc. mass & $\mathrm{pH}$ & Calc. Mass \\
\hline $0 \mathrm{~s}$ & 4.5 & $0 \mathrm{~g}$ & 4.5 & $0 \mathrm{~g}$ \\
\hline $30 \mathrm{~s}$ & 4.5 & $0 \mathrm{~g}$ & 3.5 & $1.3 \mathrm{~g}$ \\
\hline $300 \mathrm{~s}$ & 4.0 & $0.1 \mathrm{~g}$ & \multicolumn{2}{|c|}{ not measured } \\
\hline
\end{tabular}

\section{CONCLUSIONS}

This work shows that EHD enhanced transport and trapping of airborne particles to a microfluidic air-liquid interface is improved by over 130 times compared to passive transportation and trapping. Furthermore, a liquid based collector electrode is successfully used in conjunction with a corona discharge electrode surrounded by air. A stable air-liquid interface is realized by using a perforated silicon diaphragm with each pore functioning as a static Laplace valve. This opens up possibilities to integrate microfluidic analysis systems with air sampling instruments for rapid and efficient analysis of airborne particles.

\section{ACKNOWLEDGEMENT}

This work was sponsored by the Swedish Governmental Agency for Innovation Systems, the Swedish Foundation for Strategic Research, and the Swedish Research Council.

\section{REFERENCES}

[1] T. Frisk, D. Rönnholm, W. van der Wijngaart, and G Stemme., "A Micromachined Interface for Airborne Sample-to-Liquid Transfer and its Application in a Biosensor System", Lab on a Chip, vol. 6, pp. 15041509, 2006.

[2] T. Frisk, L. Eng, S. Guo, W. van der Wijngaart, and G. Stemme, "A Miniaturised Integrated QCM-based
Electronic Nose Microsystem", Proc. IEEE MEMS, pp. 417-420, 2007.

[3] A. Desai, S.-W. Lee, and Y.-C. Tai, "Air-to-Liquid MEMS Particle Sampler", Proc. IEEE MEMS, pp. 733-738, 2000.

[4] Y. Zhao, S.K. Chung, U.-C. Yi, and S.K. Cho, "Micro Particle Sampling on Microfabricated Perforated Filter Membranes", Proc. $\mu$ TAS, pp. 847-849, 2007.

[5] O. M. Stuetzer, "Ion Drag Pressure Generation", Journal of Applied Physics, Vol. 30, No. 7, 1959.

[6] A Mizuno, "Electrostatic Precipitation", IEEE Transactions on Dielectrics and Electrical Insulation, Vol. 7, No. 5, 2000.

[7] T. Yamamoto, G. Tanioka, M. Okubo, and T. Kuroki, "Water vapor desorption and adsorbent regeneration for air conditioning unit using pulsed corona plasma", Journal of Electrostatics, Vol. 65, No. 4, 2007.

[8] J. E. Bryan, "Experimental Study of Ion-drag Pumping Using Various Working Fluids", IEEE Transactions on Dielectrics and Electrical Insulation, Vol. 28, No. 4, 1991.

[9] N. E. Jewell-Larsen and E. Tran, "Design and Optimization of Electrostatic Fluid Accelerators", IEEE Transactions on Dielectrics and Electrical Insulation, Vol. 13, No. 1, 2006.

[10] B. L. Chua, Z. Li, D. T. McCormick, W. P. Shih, N. C. Tien, A. S. Wexler, D. A. Niemeier, and B. Holmen, "A Unipolar Corona Discharge Microfabricated Ionizer Structure for Gases at Atmospheric Pressure and Composition", Proc. IEEE MEMS, pp. 261-264, 2004.

[11] C.-P. Hsu, N. E. Jewell-Larsen, I. A. Krichtafovitch, S. W. Montogemery, J. T. Dibene, and A. V. Mamishev, "Miniaturization of Electrostatic Fluid Accelerators", Jounral of MEMS, Vol. 16, No. 4, 2007.

[12] C. J. Hogan Jr., M.-H. Lee, and P. Biswas, "Capture of Viral Particles in Soft X-Ray-Enhanced Corona Systems: Charge Distribution and Transport Characteristics", Aerosol Science and Technology, vol. 38, pp. 475-486, 2004. 